\title{
YACIMIENTOS CON INSECTOS FÓSILES EN ESPAÑA
}

\author{
Enrique PEÑALVER', Xavier MARTÍNEZ-DELCLÒS $S^{2}$ \\ Antonio ARILLO \\ ' Departament de Geologia, Facultat de Biologia, Universitat de València, Dr. Moliner, 50. \\ 46100 Burjassot (Valencia).e-mail: penalver@uv.es \\ ${ }^{2}$ Departament d'Estratigrafia i Paleontologia, Facultat de Geologia, Universitat de \\ Barcelona, 08071 Barcelona. e-mail: delclos@natura.geo.ub.es \\ ${ }^{3}$ Departamento de Biología Animal I (Entomología), Facultad de Biología, Universidad \\ Complutense, 28040 Madrid. e-mail: aarillo@teleline.es
}

Peñalver, E., Martínez-Delclòs, X. y Arillo, A. 1999. Yacimientos con insectos fósiles en España. [Localities with fossil insects from Spain]. Revista Española de Paleontología, 14(2), 231-245. ISSN 0213-6937.

\begin{abstract}
An overview is given of the Spanish fossil insect localities, some of them as yet unpublished. They are presented in chronostratigraphical order. A taxonomic list of the insects found at each locality is included. Localities with fossil insects are scarce in Spain and only a small number of them have been studied in any depth. A large part of the 41 sites on record are hitherto unexplored and the majority of these are merely chance findings of a handful of specimens. Prior to the 1980s, fossil insects had only been cited from 11 of these sites. Current studies are almost all concentrated on the Cretaceous and Neogene. The insects are found in various types of rocks: in coal, lithographic limestone, oil shales, diatomites, calcareous nodules, and amber. As to the type of remains to be found there are body fossils (whole specimens or broken chunks) and traces (tunnels or nests embedded in sediment or originally in organic material such as leaves or trunks). Most localities are situated in the North and East of the Iberian Peninsula.
\end{abstract}

\section{Keywords: Insects, fossil record, ichnology, Phanerozoic, Spain.}

\begin{abstract}
RESUMEN
Este artículo es una puesta al día de los yacimientos con insectos fósiles en España, algunos de los cuales son inéditos. Todos se presentan ordenados de acuerdo con su edad geológica. Para cada yacimiento se incluye una relación taxonómica de los insectos citados en la bibliografía. En España no existen muchos yacimientos con insectos fósiles y sólo se ha estudiado con detalle un pequeño número de ellos. La mayoría de las 41 localidades conocidas están todavía por muestrear ya que únicamente han proporcionado algunos ejemplares aislados. De hecho, únicamente en once yacimientos ya habían sido citados insectos fósiles en la bibliografía con anterioridad a la década de los 80 . En la actualidad, los estudios paleoentomológicos prácticamente se centran en el Cretácico y en el Neógeno. Los yacimientos en conjunto se localizan en varios tipos de substratos: en carbón, en calizas litográficas, en ritmitas bituminosas, en diatomitas, en nódulos calcáreos y en ámbar. En cuanto al tipo de restos que se hallan, comprenden restos directos (individuos completos o partes desarticuladas) y una amplia muestra icnológica -ya sean galerías o nidos en substrato inorgánico (sedimentos) u originalmente en substrato orgánico (hojas o troncos). Geográficamente los yacimientos se concentran en su mayoría en la parte norte y este de la Península Ibérica.
\end{abstract}

Palabras clave: Insectos, registro fósil, icnología, Fanerozoico, España.

\section{INTRODUCCIÓN}

Los yacimientos paleoentomológicos no son abundantes ya que para que se conserven los insectos se requieren condiciones paleoambientales muy peculiares.
Algunas de las que intervienen, ya sean de manera independiente o combinada, son: la llegada del insecto a un fondo acuático anóxico, un medio con una sedimentación rápida, la presencia de velos microbianos, un $\mathrm{pH}$ no ácido, inclusión en resina, entre otros. No 


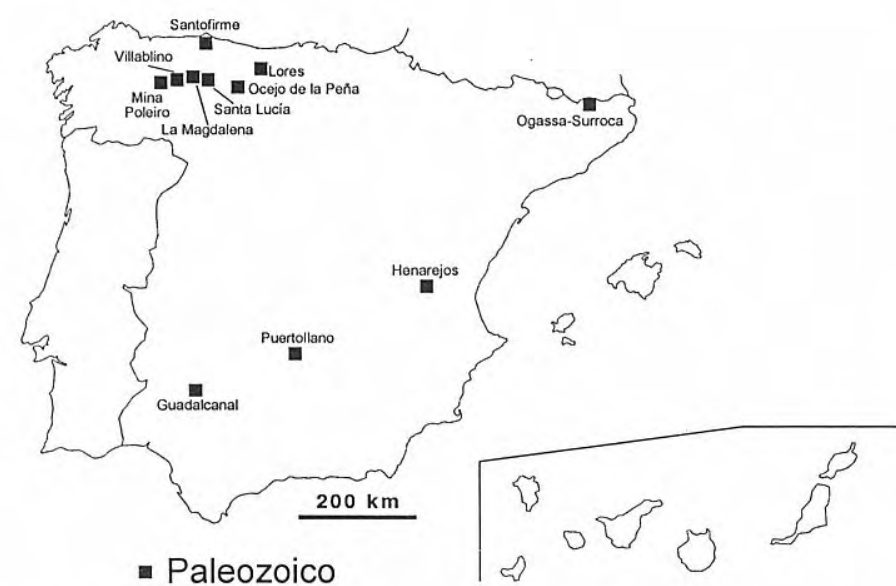

Figura 1. Localización geográfica de los yacimientos con insectos fósiles del Paleozoico en España.

obstante, en los yacimientos en los que originalmente se dieron algunas de estas características, la diversidad y la abundancia de insectos fósiles son generalmente muy grandes.

En España han sido hallados insectos fósiles o marcas de su actividad, hasta el momento, en cuarenta y un yacimientos, muchos de reciente localización (Figs. 1-3). De estos, únicamente en once de ellos -La Magdalena, Mina Poleiro y Santa Lucía en León, Izarra en Álava, Montsec y Bellver de Cerdanya en Lleida, AlcoverMontral en Tarragona, Ribesalbes en Castellón y Rubielos de Mora, Linares de Mora y Libros en Teruel-, ya habían sido citados insectos fósiles en la bibliografía con anterioridad a la década de los 80. Esto da una idea del reciente impulso que el estudio de este grupo de artrópodos ha tenido en nuestro país, aunque en la actualidad los estudios paleoentomológicos prácticamente se centran en el Cretácico y en el Neógeno.

Es de destacar que recientemente han aparecido en España cuatro yacimientos de ámbar del Cretácico Inferior. Sólo uno de ellos, el de Peñacerrada en Álava, ha librado ya cerca de 1200 restos de insectos, mientras en los otros tres yacimientos de insectos, estos se presentan de manera testimonial.
El registro fósil de insectos en nuestro país no se limita a restos de individuos completos o a partes desarticuladas, sino también a una amplia muestra icnológica. Los substratos en los que han sido hallados son muy variados y comprenden carbón, calizas litográficas, ritmitas bituminosas, diatomitas, nódulos calcáreos y ámbar.

La mayor parte de los yacimientos peninsulares se localizan en el área norte, principalmente los yacimientos del Paleozoico, y en el este, aunque existen citas de insectos fósiles en la parte sudoeste, en Mallorca y trazas de su actividad en las Islas Canarias.

\section{YACIMIENTOS CON INSECTOS FÓSILES}

Los insectos aparecen en el registro fósil en el Devónico Inferior; no obstante, los Pterigota, o insectos con alas, no aparecen hasta el Carbonífero superior (Namuriense). Los restos más antiguos hallados en España pertenecen al Carbonífero, aunque de manera esporádica e irregularmente estudiados por diversos autores. Falta por realizar una búsqueda activa de estos insectos fósiles en nuestro país y su estudio en extenso.

\section{EL REGISTRO DEL PALEOZOICO}

De las áreas donde aflora el Carbonífero en España, en siete han aparecido insectos fósiles: a) la cuenca leonesa, con los yacimientos de Ocejo de la Peña, La Magdalena, Mina Poleiro, Villablino y Santa Lucía; b) en Asturias, con el yacimiento de Santofirme; c) en Palencia, con el yacimiento de Lores; d) la cuenca gerundense, con las minas de carbón de Ogassa-Surroca; e) la conquense, con las minas de Henarejos y f) en Puertollano (Ciudad Real). Recientemente, Brauckmann (1993) ha publicado una síntesis de los insectos estudiados en el Carbonífero de España hasta ese momento. El registro del Pérmico en España es muy pobre y se limita a un único yacimiento en Guadalcanal (Sevilla) que ha proporcionado unos pocos ejemplares.

\section{Lámina I}

Insectos fósiles, o huellas de su actividad, de yacimientos españoles; todos los ejemplares figurados, a excepción de los de la figura 8, provienen de yacimientos inéditos.

1 Ala anterior de Megasecoptera del yacimiento carbonífero de Ogassa-Surroca (Girona). Escala milimétrica.

2 Élitro de coleóptero Cupedidae del Triásico de Novelda (Alicante). Barra $=2 \mathrm{~mm}$.

3 Galerías de xilófagos, 3a) en corte longitudinal (MGUV3175) y 3b) transversal (MGUV-3176), en troncos del Cretácico de Rubielos de Mora (galerías) en la provincia de Teruel. Escala centimétrica en 3 a y milimétrica en $3 b$.

4 Hemíptero pentatómido del Oligoceno de Sarral (Tarragona). Barra $=2 \mathrm{~mm}$.
5 Díptero Bibionidae del género Plecia del Mioceno de Vilobí del Penedès (Barcelona). Barra $=2 \mathrm{~mm}$.

6 Coleóptero (MGUV-3152) de la superfamilia Curculionoidea del Mioceno de Bicorp (Valencia). Barra $=2 \mathrm{~mm}$.

7 Nivel de acumulación de larvas de odonatos anisópteros (MGUV-2469) del Mioceno de la Cuenca de Sorbas (Almería). Escalas centimétricas.

8 Celdas de nidificación de himenópteros (MGUV-3103), posiblemente de la familia Anthophoridae, del Pleistoceno de Corralejo (Fuerteventura). Escala centimétrica. 
Lámina I

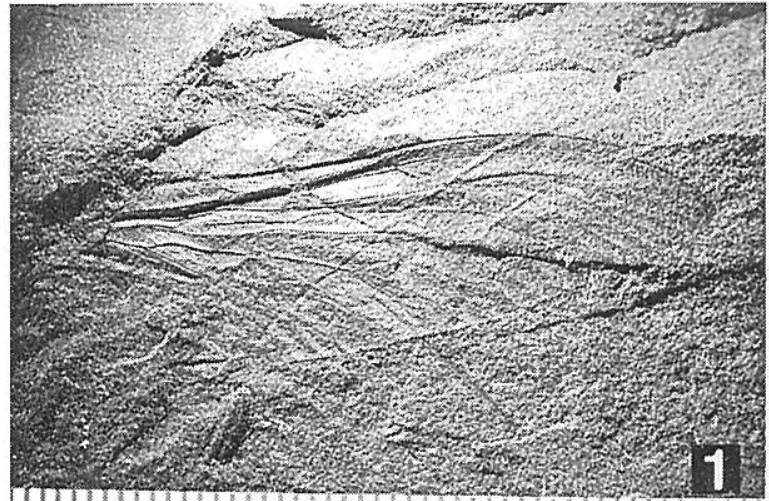

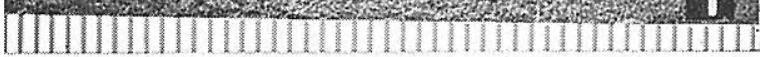

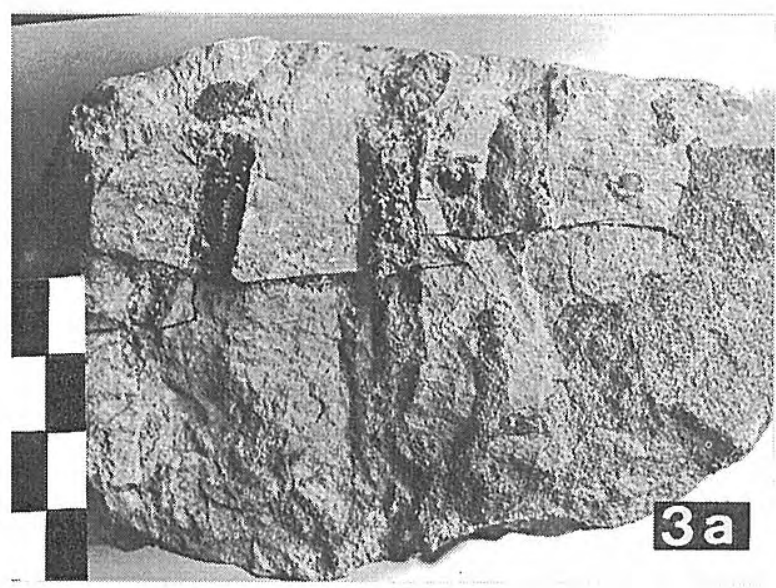

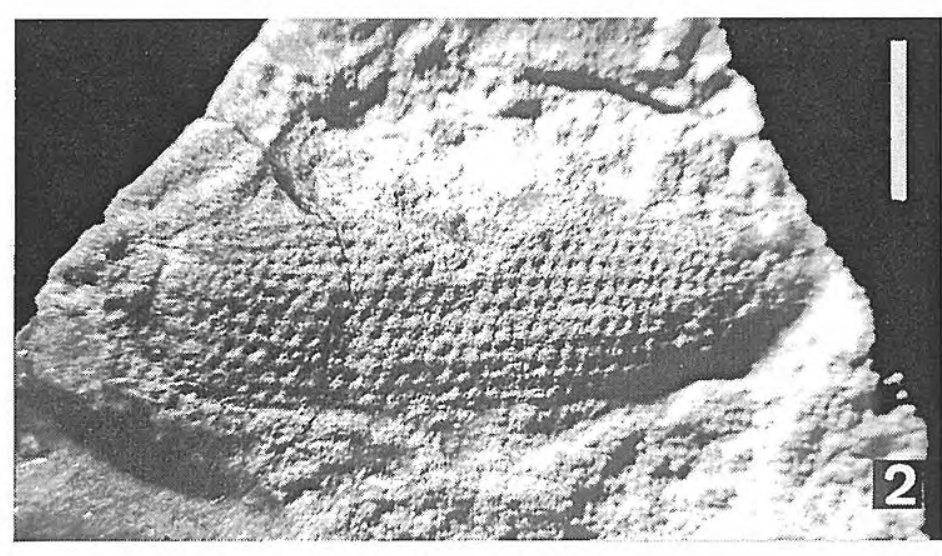

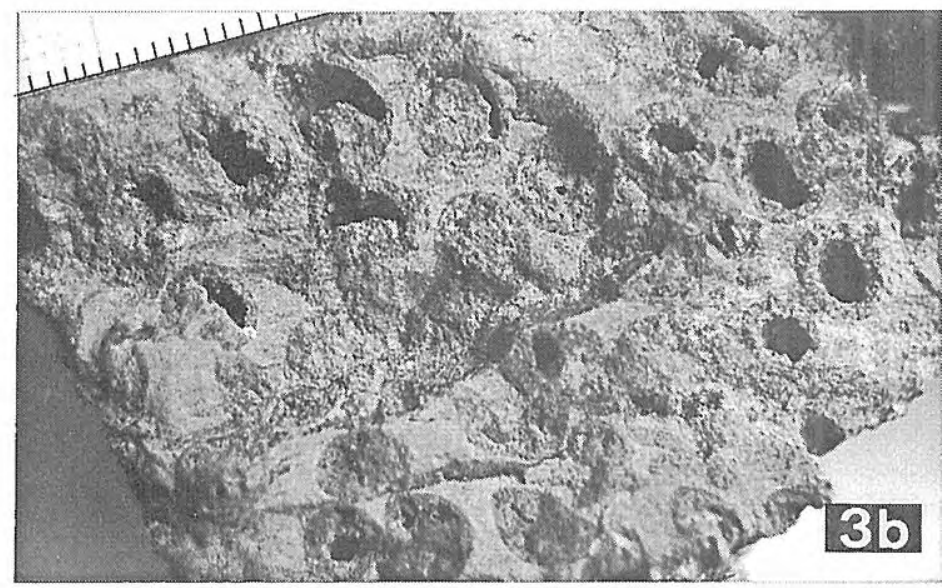

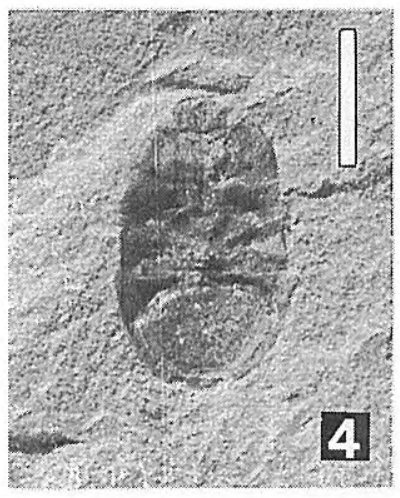
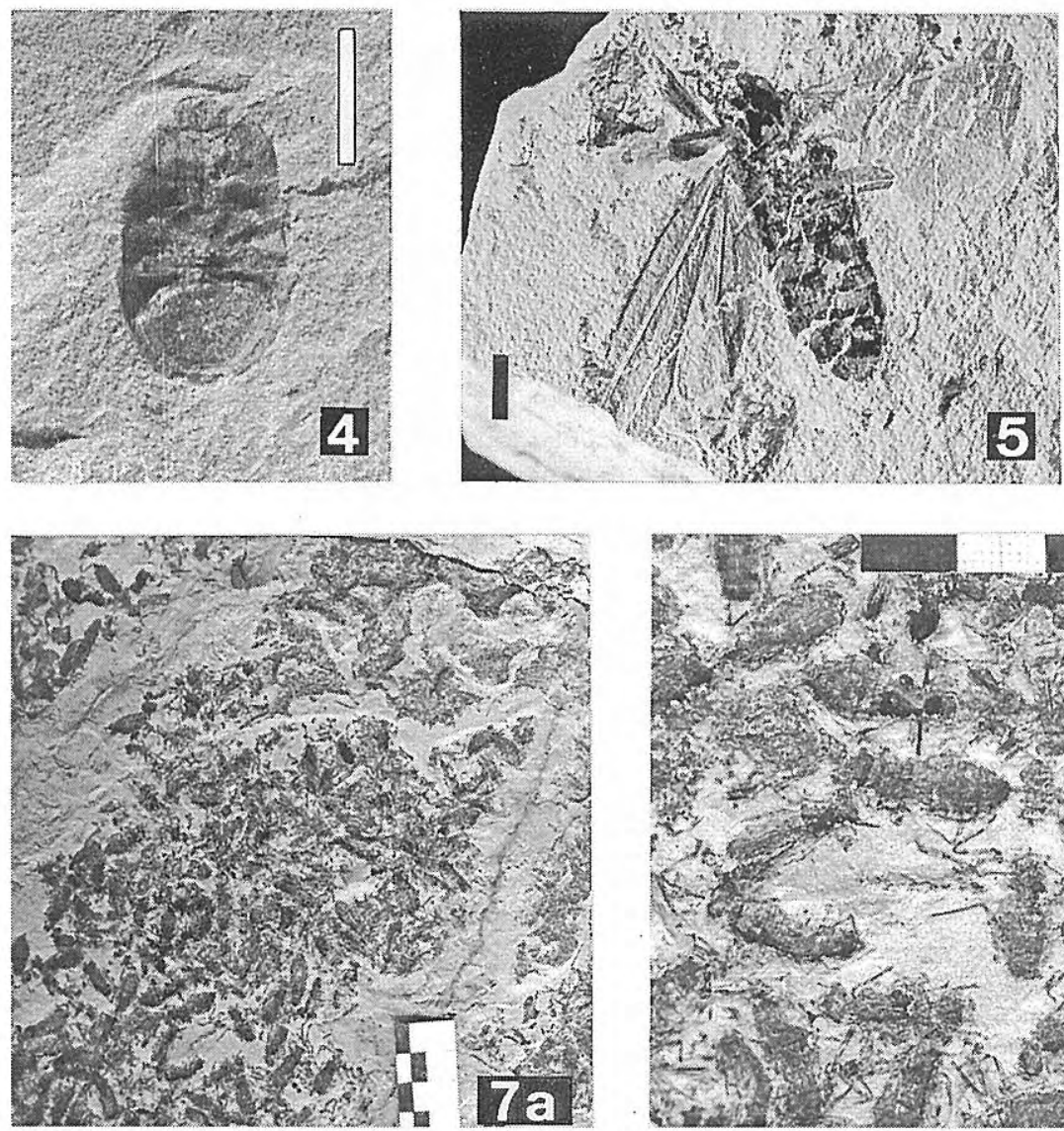
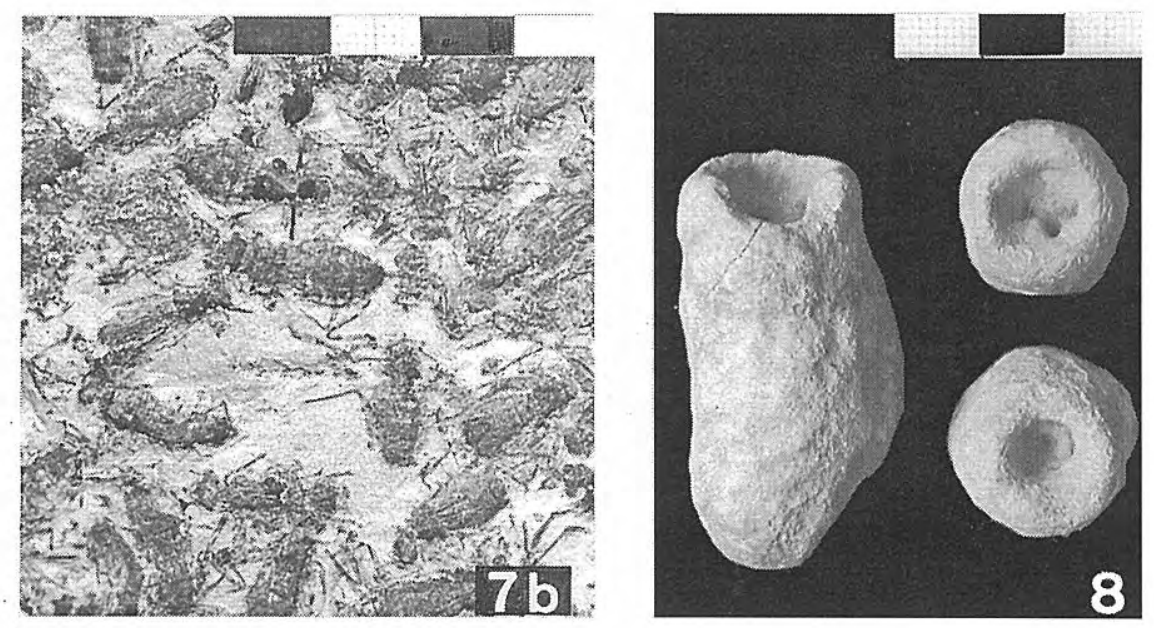

Revista Española de Paleontología, 14 (2), 1999. 


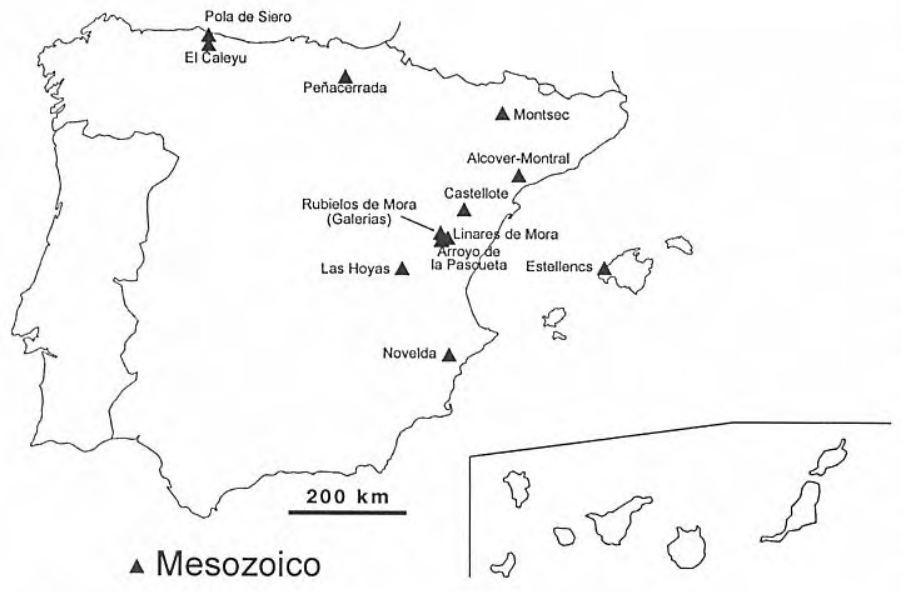

Figura 2. Localización geográfica de los yacimientos con insectos fósiles del Mesozoico en España.

\section{Carbonífero}

Westfaliense A

En la mina de carbón a cielo abierto de Santofirme, cerca de Posada de Llanera (Asturias), se localizó, a principios de los 80, un nivel muy rico en restos de insectos (E. Bernárdez, com. escrita, 1998); desgraciadamente, dicho nivel está actualmente cubierto y no se conoce el paradero exacto de los ejemplares hallados, que podrían encontrarse entre el material no catalogado, en los almacenes, del Dpto. de Geología de la Universidad de Oviedo.

\section{Westfaliense D}

En Lores, en la Pernía, al norte de Cervera de Pisuerga (Palencia), Kukalová-Peck y Brauckmann describieron, en 1992, un nuevo Protorthoptera que denominaron Cantabrala gandli Kukalová-Peck and Brauckmann (1992).

\section{Estefaniense (Cantabriense)}

A partir de un único ejemplar, Schneider (1983) cita el género Xenoblatta Handlirsch (Blattodea: Phyloblattidae) en Ocejo de la Peña (NE de León). Recientemente, un ala aislada del mismo género es figurada por Iwaniw (1985) como procedente de Santa María (Fm. Coronas), sin embargo corresponde al mismo individuo estudiado por Schneider (Wagner, com. escrita, 1997).

\section{Estefaniense B}

En la cuenca carbonífera de La Magdalena (León), Carpenter (1963) describió la nueva familia Archineuridae, incluida en el Orden Megasecoptera; la familia está formada a partir de la especie Archineura hispanica Carpenter. El ejemplar fue hallado al norte de La Magdalena, a lo largo de la carretera que parte a Barrios de Luna. En ese mismo trabajo se cita también un ala anterior de blátido de la Mina Poleiro; esta mina es de localización incierta, aunque Wagner (com. escrita, 1997) indica que podría hallarse en la zona de El Bierzo (León). Recientemente han sido citados en La Magdalena restos vegetales con trazas de alimentación y posibles agallas producidas por la actividad de insectos (Castro, 1997).

En Villablino, cerca de la Magdalena y dentro de la cuenca carbonífera leonesa, Álvarez-Ramis (1990) cita un ejemplar completo y un ala aislada de blátidos. El primero lo incluye en la especie Phyloblatta monubilis Meunier forma spathulata Bolton, y el segundo lo cita como indeterminado. Sin embargo, Phyloblatta monubilis, hallada en Commentry (Francia), había sido considerada por Schneider (1983) como sinónima de la especie Compsoblatta ovata (Meunier).

En la cuenca carbonífera catalana se encuentra el yacimiento de Ogassa-Surroca (Girona) conocido por su riqueza paleobotánica. En él se ha encontrado un ala anterior de Megasecoptera (Sinitchenkova, com. personal, 1997) (Lám. I, fig. 1). Este ejemplar se encuentra depositado en el Departament d'Estratigrafia i Paleontologia de la Universitat de Barcelona.

En la zona de Ciñera-Matallana y Mina El Oro, en la Fm. San José del Sinclinal de Matallana, al este de Santa Lucía (La Pola de Gordón, provincia de León), se encontraron varias trazas de mordeduras, posiblemente por insectos, en hojas de Neuropteris praedentata Gothan. Aquellas fueron emplazadas por Van Amerom (1966) en el nuevo icnogénero e icnoespecie Phagophytichnus ekowskii Van Amerom.

En 1970 Wagner y Artieda, en la misma zona pero en la Fm. Pastora, citan alas de insecto. Según Wagner (com. escrita, 1997), en varias cuencas mineras españolas del Westfaliense y del Estefaniense se han hallado restos de insectos que están sin estudiar. Una parte importante de ellos fueron entregados al Dr. Laurentiaux (Reims, Francia), en los años sesenta, para un estudio que nunca finalizó.

\section{Estefaniense C}

Existe una escueta cita de restos de insectos fósiles en el Estefaniense C de Puertollano, provincia de Ciudad Real, en el trabajo de Soler-Gijón (1997).

En el yacimiento del Carbonífero Superior de Henarejos (Cuenca) han sido halladas dos alas aisladas de blátidos, una anterior y otra posterior. Estos ejemplares inéditos están depositados en la Colección particular del Sr. Armando Díaz-Romeral (Cuenca).

\section{Pérmico}

En Guadalcanal (Sevilla) han sido citados dos afloramientos con insectos del Autuniense (Pérmico Inferior): Cantera de Ladrillos y Charco de la Sal. Broutin en 1986 cita estos afloramientos como del tránsito Estefaniense superior-Autuniense, pero según Wagner (com. escrita, 1997) son de edad Autuniense. Los restos de insectos corresponden a un ala de odonato de la subfamilia Oligotypinae (Pritykina, cita escrita) en Cantera de Ladrillos y a dos alas de blátidos halladas en Charco de la Sal; un ala de la familia Blattinopsidae, posiblemente del género Glaphyrophlebia (Rasnitsyn, com. personal), y un ala anterior de Spiloblattinidae (Vishniakova, cita escrita). Este material está depositado actualmente en el Instituto de Paleontología de la Academia Rusa de Ciencias, en Moscú. 


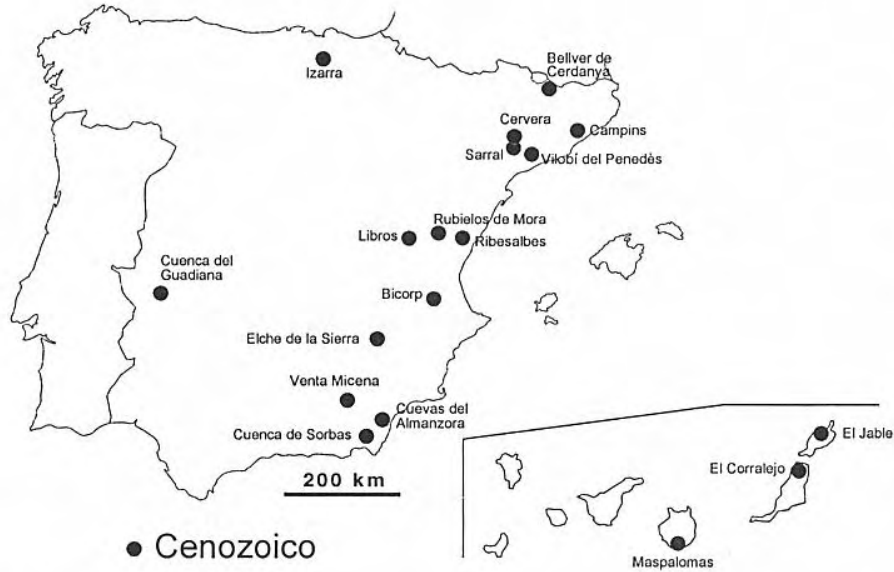

Figura 3. Localización geográfica de los yacimientos con insectos fósiles del Cenozoico en España.

\section{EL REGISTRO DEL MESOZOICO}

En los terrenos mesozoicos de España se conocen restos de insectos del Triásico y del Cretácico.

\section{Triásico}

El período Triásico cuenta con tres yacimientos: Estellencs (Mallorca), Alcover-Montral (Tarragona) y Novelda (Alicante). El registro fósil de estos yacimientos es muy escaso y prácticamente no ha sido estudiado. Se ha prestado cierta atención a los insectos fósiles de Alcover-Montral (Vía y Calzada, 1987), sin embargo, el tipo de fosilización sólo permite observar el contorno general del cuerpo, por lo que una determinación taxonómica mínimamente adecuada es imposible.

En el Triásico Medio (Anisiense) de Estellencs han sido hallados un coleóptero, un lepidóptero, larvas de efemerópteros y varios insectos indeterminados (Calafat y Sáez, 1987; Calafat, 1988). No obstante, se pone en duda la atribución de uno de los ejemplares al grupo de los lepidópteros, ya que podría tratarse de un efemeróptero adulto. Otra cita de un nuevo afloramiento en Estellencs corresponde a restos de blátidos, Ansorge (1997 y com.personal 1998).

En el Triásico Medio (Ladiniense) de AlcoverMontral se han citado un ala anterior de efémera de gran tamaño, un gerárido y un coleóptero (Vía y Calzada, 1987). El ala de efémera gigante fue determinada en 1977 por Vía et al. como perteneciente a un odonato Agrionidea. El mismo ejemplar, en 1985, fue denominado, por Vía y Calzada, Montraleon multinervium, emplazándolo en los "hemipteroideos" Mesogereonidae; no obstante, como la especie fue citada en los resúmenes de un congreso no tuvo validez taxonómica. En 1987 estos autores describen formalmente el ejemplar como Montralia muelleri y lo incluyen entre las efémeras.

El yacimiento inédito del Triásico Medio-Superior de Novelda, de facies Keuper, se localiza en el paraje denominado Font de la Reina. En éste ha sido recolectado, junto a un gran número de restos vegetales pertenecientes al orden Equisetales, un élitro de coleóptero (Lám. I, fig. 2) de la familia Cupedidae (Ponomarenko, com. personal, 1997), depositado en la colección museográfica del Grupo Cultural Paleontológico de Elche (Alicante).

\section{Cretácico}

Del Cretácico Inferior se conocen en España tres importantes áreas con yacimientos de insectos: la zona de los Montes de Cantabria en Álava, del Montsec en Lleida y la de Las Hoyas en Cuenca. En ellas aparece un abundante registro, siendo el afloramiento del Montsec, denominado La Pedrera de Rúbies, el conocido y estudiado desde más antiguo de todos los que presentan insectos fósiles en España (Meunier, 1902, 1903, 1904). Estos yacimientos del Cretácico corresponden bien a calizas laminadas (o yacimientos de compresión) o bien a depósitos de ámbar (o yacimientos de inclusión). Unas pocas localidades con ámbar del Cretácico, de reciente localización, son potencialmente muy interesantes, sin embargo no han sido estudiadas y cuentan sólo con algunos ejemplares obtenidos.

Los yacimientos paleoentomológicos del Cretácico son de gran importancia para el conocimiento de la evolución de los insectos. En este momento de la historia de la Tierra aparecen gran número de nuevas familias de insectos, similares a las actuales, coincidiendo con el período de diversificación inicial de las angiospermas. Se ha de tener en cuenta que durante el Cretácico Superior más del $80 \%$ de las familias actuales de insectos ya existían.

En el Berriasiense superior-Valanginiense inferior del Montsec (Lleida) se han estudiado, desde principios de siglo, 15 órdenes de insectos, con más de 26 especies nuevas, emplazadas muchas en géneros también nuevos (Tabla 1). Son dos los afloramientos que se conocen: La Pedrera de Rúbies, también llamado La Pedrera de Meià o La Pedrera de Santa Maria de Meià, y La Cabrua. En el Barremiense de Las Hoyas (provincia de Cuenca) se conocen 14 órdenes de insectos (Tabla 2). Ambos yacimientos son ampliamente conocidos en la bibliografía, no obstante conviene destacar que si bien los órdenes de insectos que aparecen son prácticamente los mismos, la abundancia de individuos y las familias representadas son bastante diferentes.

Un importante yacimiento de ámbar del Aptiense ha sido recientemente hallado en los Montes de Cantabria (Álava). Son dos los afloramientos excavados y han sido denominados Peñacerrada I y II. Presentan un gran número de inclusiones de origen orgánico que actualmente están siendo estudiadas por un equipo pluridisciplinar dirigido desde el Museo de Ciencias Naturales de Vitoria (Alonso et al., en prensa). Los insectos son el grupo más numeroso e incluyen representantes de los órdenes Thysanura, Blattodea, Psocoptera, Thysanoptera, Orthoptera, Hemiptera, Neuroptera, Trichoptera, Lepidoptera, Coleoptera, Diptera e Hymenoptera (Arillo, 1998; Ortuño, 1998 y Martínez-Delclòs et al., 1998). 


\begin{tabular}{|c|c|c|}
\hline ORDEN & Familia & Género y especie \\
\hline \multirow[t]{5}{*}{ EFEMEROPTERA } & Pothamantidae & Mesopalingea lerida $^{(1 \text { y2) }}$ \\
\hline & Euthyplociidae & Euthyplociidae gen. et sp. nov. ${ }^{(2)}$ \\
\hline & Leptophlebiidae & Leptophlebiidae gen. et sp. nov. ${ }^{(2)}$ \\
\hline & Indet. 1 & Gen. et sp. nov. ${ }^{(2)}$ \\
\hline & Indet. 2 & Gen. et sp. nov. ${ }^{(2)}$ \\
\hline \multirow[t]{7}{*}{ ODONATA } & Aeshnidae? & Palaeaeschna vidali ${ }^{(2,3,4 y 5)}$ \\
\hline & \multirow[t]{2}{*}{ Aeschnidiidae } & Lleidoaeschnidium valloryi ${ }^{(6)}$ \\
\hline & & Gen. et sp. indet. ${ }^{(6)}$ \\
\hline & Gomphidae & Ilerdaegomphus pallerolae ${ }^{(7 \text { y } 29)}$ \\
\hline & Libellulidae? & Condalia woottoni ${ }^{(1 \text { y } 2)}$ \\
\hline & Indet. 1 & Samarura sp. ${ }^{(8)}$ \\
\hline & Indet. 2 & Gen. et sp. indet. ${ }^{(8)}$ \\
\hline \multirow[t]{7}{*}{ BLATTODEA } & \multirow[t]{4}{*}{ Mesoblattinidae } & Mesoblattina colominasi ${ }^{(3 \mathrm{y} 9)}$ \\
\hline & & Artitocoblatta hispanica $^{(1 \text { y9) }}$ \\
\hline & & Nogueroblatta fontllongae $e^{(9)}$ \\
\hline & & N. nana ${ }^{(9)}$ \\
\hline & Polyphagidae & Cretaholocompsa montsecana ${ }^{(9)}$ \\
\hline & Blattulidae & Orbablattula infrequens ${ }^{(9)}$ \\
\hline & Corydiidae & Eythyrrhaphinae gen. et sp. nov. ${ }^{(10)}$ \\
\hline ISOPTERA & Hodotermitidae & Meiatermes bertrani ${ }^{(11 \mathrm{y} 12)}$ \\
\hline FASMOPTERA? & Chresmodidae & Chresmoda aquatica $^{(2 \mathrm{y} 13)}$ \\
\hline \multirow[t]{8}{*}{ HYMENOPTERA } & \multirow[t]{2}{*}{ Ephialtitidae } & Ephialtites jurassicus ${ }^{(4,23,24 y 30)}$ \\
\hline & & Ephialtitidae nov. sp. ${ }^{(25)}$ \\
\hline & \multirow[t]{2}{*}{ Gasteruptiidae } & Manlaya nov. sp. ${ }^{(10)}$ \\
\hline & & Gasteruptiidae gen. nov. ${ }^{(25)}$ \\
\hline & \multirow[t]{2}{*}{ Sphecidae } & Cretosphex catalaunicus ${ }^{(26)}$ \\
\hline & & ? Archisphex nov. sp. ${ }^{(10)}$ \\
\hline & Sapygidae & Sapyginae gen. indet. sp. nov. ${ }^{(10)}$ \\
\hline & Anaxyelidae & Syntexinae sp. nov. ${ }^{(25)}$ \\
\hline TRICHOPTERA & Indet. & Fam. indet. ${ }^{(10)}$ \\
\hline \multirow[t]{7}{*}{ DIPTERA } & Bibionidae & Bibionidae gen. et sp. nov. ${ }^{(10)}$ \\
\hline & Rhagionidae & Rhagionidae gen. et sp. nov. ${ }^{(10)}$ \\
\hline & Nemestrinidae & Gen. et sp. indet. ${ }^{(27)}$ \\
\hline & Tipulidae & Gen, et sp. indet. ${ }^{(27)}$ \\
\hline & Mycetophilidae & Gen. et sp. indet. ${ }^{(27)}$ \\
\hline & Sciomyzidae & Gen. et sp. indet. (larva) ${ }^{(1)}$ \\
\hline & Stratiomyiidae & Gen. et sp. indet. (larva) ${ }^{(1)}$ \\
\hline
\end{tabular}

\begin{tabular}{|c|c|c|}
\hline \multirow[t]{4}{*}{ ORTHOPTERA } & Locustopsidae & Zeunerella sp. ${ }^{(10 y 14)}$ \\
\hline & Haglidae & Gen. et sp. nov. ${ }^{(2)}$ \\
\hline & Elcanidae & Gen. et sp. nov. ${ }^{(2)}$ \\
\hline & Locustopseidae? & Gen. et sp. nov. ${ }^{(2)}$ \\
\hline \multirow[t]{12}{*}{ HEMIPTERA } & \multirow[t]{4}{*}{ Palaeontinidae } & Pachypsyche vidali $(2,4,15$ y 16) \\
\hline & & Ilerdocossus villaltai $(2,4 \mathrm{y} 17)$ \\
\hline & & Wonnacottella pulcherrima ${ }^{(1,2,4 y 18)}$ \\
\hline & & Montsecocossus sirerai ${ }^{(2,4 \text { y } 16)}$ \\
\hline & Cicadellidae & Acocephalites breddini ${ }^{(4,16 \text { y } 19)}$ \\
\hline & \multirow[t]{2}{*}{ Procercopidae? } & Myangadina sp. nov. ${ }^{(10)}$ \\
\hline & & Gen. et sp. indet. ${ }^{(10)}$ \\
\hline & Cixiidae & Cixiidae gen. et sp. nov. ${ }^{(2)}$ \\
\hline & Progonocimicidae & Progonocimicidae gen. et sp. nov. ${ }^{(10)}$ \\
\hline & Indet. & Aleyrodoidea? gen. et sp. indet. ${ }^{(1)}$ \\
\hline & Indet. & Geocorisae gen. et sp. indet. ${ }^{(1)}$ \\
\hline & Belostomatidae & Iberonepa romerali ${ }^{(20)}$ \\
\hline RAPHIDIOPTERA & Mesoraphidiidae & ? Mesoraphidia sp. ${ }^{(10 \mathrm{y} 31)}$ \\
\hline NEUROPTERA & Chysopidae & Gen. et sp. indet. ${ }^{(10)}$ \\
\hline \multirow[t]{5}{*}{ COLEOPTERA } & Buprestidae & Pseudochrysobothris ballae ${ }^{(1 \mathrm{y} 21)}$ \\
\hline & Scarabaeidae & Antemnacrassa monreali ${ }^{(16 \text { y } 22)}$ \\
\hline & Belidae & Montsecbelus solutus ${ }^{(1,16,22 \text { y } 28)}$ \\
\hline & Eccoptarthridae & Jarzembowskia edmundi ${ }^{(28)}$ \\
\hline & Indet. & Brevispinnatus paludis ${ }^{(16 \mathrm{y} 22)}$ \\
\hline
\end{tabular}

Tabla 1. Taxones de insectos hallados, hasta el momento, en los yacimientos del Cretácico Inferior del Montsec (Lleida). 1) Whalley y Jarzembowski (1985); 2) Martínez-Delclòs (1991b); 3) Meunier (1914); 4) Martínez-Delclòs (1990); 5) Nel et al. (1994); 6) Nel y Martínez-Delclòs (1993a); 7) MartínezDelclòs y Nel (1994); 8) Nel y Martínez-Delclòs (1993b); 9) Martínez-Delclòs (1993); 10) Ansorge (1991a); 11) Lacasa y Martínez-Delclòs (1986b); 12) Martínez-Delclòs y Martinell (1995); 13) Martínez-Delclòs (1989a); 14) Ansorge (1991b); 15) Meunier (1902); 16) Martínez-Delclòs (1991a); 17) Gómez (1984); 18) Martínez-Delclòs y Nel (1995a); 19) Meunier (1904); 20) Martínez-Delclòs et al. (1995); 21) Alekseev (1993); 22) Gómez (1979); 23) Meunier (1903); 24) Rasnitsyn (1975); 25) Rasnitsyn (com.personal, 1997); 26) Ansorge (1993); 27) Lacasa y Martínez-Delclòs (1986a); 28) Zherikhin y Gratshev (1997); 29) MartínezDelclòs (1989b); 30) Rasnitzyn (1975) y 31) Gómez (1986). 


\begin{tabular}{|c|c|c|}
\hline ORDEN & Familia & Género y especie \\
\hline \multirow[t]{2}{*}{ EFEMEROPTERA } & \multirow[t]{2}{*}{ Leptophlebiidae } & Gen. et sp. nov. 1 (larva) ${ }^{(1,2 y 10)}$ \\
\hline & & Gen. et sp. nov. 2 (larva) ${ }^{(1 \text { y } 2)}$ \\
\hline \multirow[t]{6}{*}{ ODONATA } & Aeshnidae & 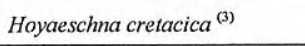 \\
\hline & Gomphidae & Ilerdaegomphus torcae ${ }^{(4)}$ \\
\hline & \multirow[t]{4}{*}{ Aeschnidiidae } & Iberoaeschnidium conquensis ${ }^{(5)}$ \\
\hline & & Gigantoaeschnidium ibericus $^{(\mathrm{s})}$ \\
\hline & & Nannoaeschnidium pumilio ${ }^{(5)}$ \\
\hline & & Gen. et sp. indet. ${ }^{(3)}$ \\
\hline \multirow[t]{3}{*}{ BLATTODEA } & \multirow[t]{3}{*}{ Mesoblattinidae } & Hispanoblatta sumptuosa ${ }^{(6)}$ \\
\hline & & Nogueroblatta fontllongae ${ }^{(6)}$ \\
\hline & & Mesoblattinidae forma $\mathrm{C}^{(2)}$ \\
\hline ISOPTERA & Hodotermitidae & Meiatermes bertrani ${ }^{(7)}$ \\
\hline FASMOPTERA? & Chresmodidae & Chresmoda sp. ${ }^{(8)}$ \\
\hline \multirow[t]{2}{*}{ ORTHOPTERA } & \multirow[t]{2}{*}{ Gryllidae } & Gen. et sp. nov. $1^{(1 \text { y } 2)}$ \\
\hline & & Gen. et sp. nov. $2^{(1 \text { y } 2)}$ \\
\hline \multirow[t]{4}{*}{ HEMIPTERA } & Cixiidae? & Fulgoroidea forma $\mathrm{A}^{(2)}$ \\
\hline & \multirow[t]{3}{*}{ Belostomatidae } & Iberonepa romerali ${ }^{(9)}$ \\
\hline & & Gen. et sp. nov. ${ }^{\left(1 y_{2}\right)}$ \\
\hline & & Gen. et sp. nov. ${ }^{\left(1 y^{2}\right)}$ \\
\hline
\end{tabular}

Otro yacimiento análogo al anterior se encuentra en Rubielos de Mora y ha sido nominado como Arroyo de la Pascueta. Es también de ámbar del Cretácico Inferior (Albiense inferior-medio) y se localiza en los niveles lignitosos de la Fm. Arenas de Utrillas, en forma de varios afloramientos. En este ámbar de color rojo muy oscuro se han localizado recientemente restos de un díptero y un ejemplar completo de homóptero, siendo buenas las expectativas de reunir, en futuras excavaciones, una colección de ejemplares. Por último, Boscá (1910) menciona la posible existencia de insectos fosilizados en grandes trozos de ámbar cretácico de color negruzco-rojizo en Linares de Mora, población cercana a Rubielos de Mora, en la provincia de Teruel.

En la base de la Fm. Ullaga (Albiense superior) de Pola de Siero (Asturias) ha sido hallado un fragmento de ala, que recuerda a un "ortopteroideo", en una pieza de ámbar. Dicho ejemplar fue hallado por E. Bernárdez y se encuentra en el Departamento de Geología de la Universidad de Oviedo (Arbizu et al., 1998). En las calizas limosas de la misma formación, pero cerca de El Caleyu (Asturias) han sido recolectados, por Miguel Ángel Prieto, varios restos de insectos incluidos en ámbar que se conservan en su colección privada. Uno de estos insectos corresponde a un ejemplar hembra de la superfamilia Empidoidea (Diptera) en perfecto estado.

En el Albiense de Castellote (Teruel) han sido citadas

\begin{tabular}{|c|c|c|}
\hline RAPHIDIOPTERA & Mesoraphidiidae & Gen. et sp. indet. $\left.{ }^{(7 y} 8\right)$ \\
\hline \multirow[t]{3}{*}{ NEUROPTERA } & Chrysopidae & Gen. et sp. nov. $1-6^{(8)}$ \\
\hline & Nymphitidae & Gen. et sp. indet. ${ }^{(7 y 8)}$ \\
\hline & Kalligrammatidae & Gen. et sp. nov. $\left.{ }^{(7 y} y^{8}\right)$ \\
\hline \multirow[t]{4}{*}{ COLEOPTERA } & Cupedidae & Gen. et sp. indet. ${ }^{(7 y 8)}$ \\
\hline & Scarabaeidae & Gen. et sp. indet. ${ }^{(7 y 8)}$ \\
\hline & Ademosynidae? & Gen. et sp. indet. ${ }^{(7 y 8)}$ \\
\hline & Indet. & Staphylinioidea gen. et sp. indet. ${ }^{(7 y 8)}$ \\
\hline HYMENOPTERA & Indet. & Apocrita gen. et sp. indet. ${ }^{(7 y 8)}$ \\
\hline MECOPTERA & Panorpidae & Orthophlebiinae sp. nov. ${ }^{(7 y 8)}$ \\
\hline \multirow[t]{2}{*}{ DIPTERA } & Nemestrinidae & Gen. et sp. indet. ${ }^{(7 y 8)}$ \\
\hline & Stratiomyiidae & Gen. et sp. indet. ${ }^{(7 y 8)}$ \\
\hline
\end{tabular}

Tabla 2. Taxones de insectos en el registro del Cretácico Inferior de Las Hoyas (Cuenca). 1) MartínezDelclòs (1991b); 2) Martínez-Delclòs (en prensa); 3) Nel y Martínez-Delclòs (1993b); 4) MartínezDelclòs y Nel (1994); 5) Nel y Martínez-Delciòs (1993a); 6) Martínez-Delclòs (1993); 7) MartínezDelclòs (1989c); 8) Martínez-Delclòs y Nel (1995b); 9) Martínez-Delclòs et al. (1995) y 10) Martínez-Delclòs y Ruiz de Loizaga (1993).

galerías de posibles larvas de insectos xilófagos en troncos fósiles (Díez et al., 1996). Estas mismas galerías han sido encontradas por uno de los autores (E.P.) en troncos fósiles del Aptiense-Albiense de Rubielos de Mora, yacimiento que llamaremos Rubielos de Mora (galerías); dos muestras están depositadas en el Museo del Departamento de Geología de la Universitat de València (Lám. I, fig. 3). Ambos yacimientos se encuentran en la Fm. Arenas de Utrillas.

\section{EL REGISTRO DEL CENOZOICO}

Se conocen, en España, restos fósiles de insectos en rocas del Oligoceno, Mioceno, Plioceno y Pleistoceno.

\section{Oligoceno}

Del Oligoceno se conocen insectos en los yacimientos de Cervera (Lleida), Campins (Barcelona) y Sarral (Tarragona). El descubrimiento de los mismos es relativamente reciente, por lo que su estudio se encuentra en los inicios. Es especialmente interesante el conocimiento de los insectos fósiles del Oligoceno español para una comparación con el rico registro fósil de este período en Europa, especialmente en Francia.

Si bien la primera cita de insectos en Cervera es de Martínez-Delclòs (1988), no es hasta 1991 cuando Martínez-Delclòs y Nel los estudian. Han aparecido 


\begin{tabular}{|c|c|c|}
\hline ORDEN & Familia & Género y especie \\
\hline \multirow[t]{5}{*}{ ODONATA } & Lestidae? & ?Lestes ${ }^{(1)}$ \\
\hline & Coenagrionidae? & Hispanocoenagrion inexpectum ${ }^{(1)}$ \\
\hline & Lindeniidae & Gen. et sp. indet. ${ }^{(1)}$ \\
\hline & \multirow[t]{2}{*}{ Libellulidae } & Gen. et sp. indet. (larva) ${ }^{(2)}$ \\
\hline & & Trameinae gen. et sp. indet. ${ }^{(3)}$ \\
\hline ISOPTERA & Mastotermitidae & Mastotermes sp. ${ }^{(4)}$ \\
\hline ORTHOPTERA & Acrididae & Gen. et sp. indet. ${ }^{(5)}$ \\
\hline \multirow[t]{8}{*}{ HEMIPTERA } & Psyllidae & Gen. et sp. indet. ${ }^{(6)}$ \\
\hline & Indet. & Aphidoidea fam. indet. ${ }^{(6)}$ \\
\hline & \multirow[t]{3}{*}{ Lygaeidae } & Geocoris monserrati $^{(7)}$ \\
\hline & & Rhyparochrominae gen. et sp. indet. ${ }^{(8)}$ \\
\hline & & Ischnodemus sp. ${ }^{(8)}$ \\
\hline & Cydnidae & Gen. et sp. indet. ${ }^{(8)}$ \\
\hline & Tingidae & Gen. et sp. indet. ${ }^{(6)}$ \\
\hline & Pentatomidae & Gen. et sp. indet. ${ }^{(6)}$ \\
\hline THYSANOPTERA & Indet. & Fam. indet. ${ }^{(9)}$ \\
\hline
\end{tabular}

\begin{tabular}{|c|c|c|}
\hline \multirow[t]{8}{*}{ COLEOPTERA } & Dityscidae & $\begin{array}{l}\text { Colymbetinae gen. et sp. indet.(larva) } \\
\text { (10) }\end{array}$ \\
\hline & Staphylinidae & Gen. et sp. indet. ${ }^{(6)}$ \\
\hline & Anthicidae & Anthicus neli ${ }^{(11)}$ \\
\hline & Anobiidae & Ernobius alonsoi ${ }^{(12)}$ \\
\hline & Latridiidae & Gen. et sp. indet. ${ }^{(6)}$ \\
\hline & Chrysomelidae & Halticinae gen. et sp. indet. ${ }^{(11)}$ \\
\hline & Curculionidae & Gen. et sp. indet. ${ }^{(6)}$ \\
\hline & Scolytidae & Gen. et sp. indet. ${ }^{(6)}$ \\
\hline \multirow[t]{3}{*}{ HYMENOPTERA } & Apidae & Apis aequisextiana $^{(13)}$ \\
\hline & Rhophitidae & Gen. et sp. indet. ${ }^{(13)}$ \\
\hline & Formicidae & Gen. et sp. indet. ${ }^{(6)}$ \\
\hline TRICHOPTERA & Indet. & Fam. indet. ${ }^{(6)}$ \\
\hline LEPIDOPTERA & Indet. & Fam. indet. ${ }^{(9)}$ \\
\hline \multirow[t]{2}{*}{ DIPTERA } & Tipulidae & Gen. et sp. indet. ${ }^{(6)}$ \\
\hline & Limoniidae? & Gen. et sp. indet. ${ }^{(6)}$ \\
\hline
\end{tabular}

Tabla 3. Insectos del Mioceno Inferior de Izarra (Álava). 1) Nel et al. (1997); 2) Arillo (1994); 3) Nel et al. (1995); 4) Nel y Arillo (1995); 5) Arillo y Ortuño (1997b); 6) Barrón et al. (1997); 7) Ortuño y Arillo (1997c); 8) Ortuño y Arillo (1997b); 9) Ortuño et al. (1996); 10) Ortuño y Arillo (1997d); 11) Arillo y Ortuño (1997a); 12) Ortuño y Arillo (1997a) y 13) Arillo et al. (1996).

solamente tres alas aisladas de odonatos: un probable Lestes, un probable Aeshna y un Aeshnidae indeterminado; también citan un élitro de coleóptero Curculionidae, posiblemente del género Hipporhinus.

En las facies carbonatadas de origen lagunar profundo de Campins, se ha citado un ala aislada de coleóptero (Anadón, 1973), junto con registro de flora.

En las facies limosas de la Fm. Sarral, en las inmediaciones del pueblo que da nombre a la formación, interestratificados entre los niveles de yesos, han aparecido varios insectos, como coleópteros, un heteróptero Pentatomidae y abundantes ejemplares de posibles heterópteros, todos por estudiar (Lám. I, fig. 4). Este material esta depositado en el Museu Comarcal de la Conca de Barberà-Centre d'Història Natural, en Montblanc (Tarragona) y en el Departament d'Estratigrafia i Paleontontologia de la Universitat de Barcelona.

\section{Mioceno}

Del Mioceno se conocen los yacimientos de Izarra en Álava, Bellver de Cerdanya en Lleida, Vilobí del Penedès en Barcelona, Ribesalbes en Castellón, Rubielos de Mora y Libros en Teruel, Elche de la Sierra en Albacete, Bicorp en Valencia, Cuenca de Sorbas en Almería y Cuenca del Guadiana en Badajoz.
El conocimiento de la riqueza paleoentomológica de estos yacimientos es muy desigual, ya que los insectos fósiles de Vilobí del Penedès, de Bicorp, de Libros, de Elche de la Sierra, de la Cuenca de Sorbas y sus trazas encontradas en la Cuenca del Guadiana, permanecen prácticamente desconocidos.

La presencia de insectos fósiles en el yacimiento del Mioceno Inferior de Izarra fue dada a conocer por Fernández-Marrón et al. (1979). Actualmente, está siendo estudiado el amplio registro paleoentomológico de este yacimiento, datado hasta hace poco como del Oligoceno (Barrón et al., 1997). La lista de grupos de insectos hallados, que incluye ejemplares de géneros poco comunes en el registro fósil mundial, se puede consultar en la Tabla 3. Este yacimiento es localidad tipo de las siguientes especies: Hispanocoenagrion inexpectum $\mathrm{Nel}$, Arillo y Ortuño, 1997 (Odonata), Geocoris monserrati Ortuño y Arillo, 1997 (Hemiptera), y Anthicus neli Arillo y Ortuño, 1997 y Ernobius alonsoi Arillo y Ortuño, 1997, entre los Coleoptera.

En el Mioceno Inferior de Vilobí del Penedès, interestratificado en los niveles de yesos que explota la compañía Vilovigyps SA, apareció a finales de los ochenta un nivel limoso con abundantes peces y donde se recolectaron dos dípteros del género Plecia (Lám. I, fig. 5). 


\begin{tabular}{|c|c|c|}
\hline ORDEN & Familia & Género y especie \\
\hline \multirow[t]{7}{*}{ ODONATA } & \multirow[t]{2}{*}{ Aeshnidae } & Oligaeschna saurai ${ }^{(1)}$ \\
\hline & & Gen. et sp. indet (larva) ${ }^{(1)}$ \\
\hline & \multirow[t]{4}{*}{ Lestidae } & Sympecma? ribesalbesensis ${ }^{(1)}$ \\
\hline & & Lestes sp. ${ }^{(1)}$ \\
\hline & & cf. Lestes sp. ${ }^{(1)}$ \\
\hline & & Gen. et sp. indet. (larva) ${ }^{(1)}$ \\
\hline & Indet. & Platycnemis? cincuneguii ${ }^{(1,2,3)}$ \\
\hline ISOPTERA & Hodotermitidae & Ulmeriella sp. ${ }^{(1)}$ \\
\hline GRYLLOPTERA & Tettigoniidae & Tettigonia aff. veridissima ${ }^{(1)}$ \\
\hline ORTHOPTERA & Acrididae & Oedipodinae gen. et sp. indet. ${ }^{(1)}$ \\
\hline PSOCOPTERA & Mesopsocidae & cf. Mesopsocus sp. (s.1.) ${ }^{(1)}$ \\
\hline \multirow[t]{9}{*}{ HEMIPTERA } & Psyllidae & Gen. et sp. indet. ${ }^{(1)}$ \\
\hline & Aphididae & Gen. et sp. indet. ${ }^{(1)}$ \\
\hline & Cercopidae & Aphrophora sp. ${ }^{(1)}$ \\
\hline & Notonectidae & Notonecta sp. ${ }^{(1)}$ \\
\hline & Nepidae & Nepa sp. ${ }^{(1)}$ \\
\hline & Anthocoridae & Gen. et sp. indet. ${ }^{(1)}$ \\
\hline & Miridae & Gen. et sp. indet. ${ }^{(1)}$ \\
\hline & Berytidae & Gen. et sp. indet. ${ }^{(1)}$ \\
\hline & Lygaeidae & cf. Lygaeidae gen. et sp. indet. ${ }^{(1)}$ \\
\hline
\end{tabular}

\begin{tabular}{|c|c|c|}
\hline THYSANOPTERA & Thripidae & Gen. et sp. indet. ${ }^{(1)}$ \\
\hline NEUROPTERA & Chrysopidae & Pronothochrysa vivesi ${ }^{(4)}$ \\
\hline COLEOPTERA & Indet. & Fam. indet. ${ }^{(2,5)}$ \\
\hline \multirow[t]{5}{*}{ HYMENOPTERA } & \multirow[t]{2}{*}{ Braconidae } & Braconidae? ${ }^{(2)}$ \\
\hline & & Helconinae gen. et sp. indet. ${ }^{(5)}$ \\
\hline & Ichneumonidae & Ophioninae gen. et sp. indet. ${ }^{(5)}$ \\
\hline & Sphecidae & Gen. et sp. indet. ${ }^{(5)}$ \\
\hline & Formicidae & Gen. et sp. indet. ${ }^{(6)}$ \\
\hline TRICHOPTERA & Phryganeidae & Phryganea sp. ${ }^{(5)}$ \\
\hline LEPIDOPTERA & Indet. & Gen. et sp. indet. ${ }^{(7)}$ \\
\hline \multirow[t]{10}{*}{ DIPTERA } & Tipulidae & Tipula sp. ${ }^{(5)}$ \\
\hline & Empididae & Hilara royoi ${ }^{(2)}$ \\
\hline & \multirow[t]{2}{*}{ Bibionidae } & Bibio sp. ${ }^{(6)}$ \\
\hline & & Penthetria sp. ${ }^{(8)}$ \\
\hline & Mycetophilidae & Gen. et sp. indet. ${ }^{(5)}$ \\
\hline & Anisopodidae & Sylvicola sp. ${ }^{(5)}$ \\
\hline & Culicidae & Gen. et sp. indet. (pupas) \\
\hline & Chironomidae & Nomochirus sampelayoi ${ }^{(2,9)}$ \\
\hline & Stratiomyidae & Odontomyia sp. (larvas) ${ }^{(10)}$ \\
\hline & Phoridae & Puliciphora 0 Aneurina sp. ${ }^{(5)}$ \\
\hline
\end{tabular}

Tabla 4. Insectos en el registro del Mioceno Inferior de Ribesalbes (Castellón). 1) Peñalver et al. (1996); 2) Gil (1926); 3) Compte (1995); 4) Peñalver et al. (1995); 5) Peñalver y Martínez-Delclòs (1993); 6) Vigón (1980); 7) Peñalver y Martínez-Delclòs (1997); 8) Peñalver (1996c); 9) Peñalver y Martínez-Delclòs (1996) y 10) Peñalver (1996a).

En el Mioceno Inferior de La Rinconada, en Ribesalbes, ha sido hallado desde el primer cuarto de siglo un número considerable de insectos fósiles. Hasta la actualidad se han descrito seis especies a partir de ejemplares de este yacimiento (dos de ellas son también géneros nuevos: Nomochirus y Pronothochrysa). En la Tabla 4 se muestra el conjunto de insectos determinados; no se incluyen algunos de los géneros y especies citados por Vigón (1980 y 1981) al haberse comprobado, durante la revisión del material, que dichas determinaciones son incorrectas.

En sedimentos del Mioceno Inferior (Aragoniense inferior) de Rubielos de Mora se han localizado cinco afloramientos con una gran cantidad de insectos: Río Rubielos, Barranco de Aguarroya, Alto de la Venta, Barranco Casas, El Bolaje de Abajo y Cerro del Porpol.

El afloramiento de Río Rubielos es la localidad tipo de la especie Zygaena? turolensis (Lepidoptera: Zygaenidae), descrita por Fernández-Rubio et al. (1991). Otro ejemplar de la misma especie es estudiado por
Fernández-Rubio y Peñalver en 1994. Los otros órdenes de insectos hallados hasta la fecha son: Odonata, Dermaptera, Orthoptera, Hemiptera, Thysanoptera, Raphidioptera, Coleoptera, Hymenoptera, Trichoptera y Diptera. La lista de familias y géneros hallados es muy elevada y pueden consultarse en Montoya et al. (1996), en donde se encuentra una recopilación bibliográfica referida a los insectos fósiles del yacimiento. La lista de los grupos de insectos hallados en Rubielos de Mora se ha visto incrementada posteriormente con las citas de las familias Greenideidae (Hemiptera), Phlaeothripidae (Thysanoptera), Chrysomelidae (Coleoptera), Bethylidae (Hymenoptera) y Sciaridae (Diptera) con el género Sciara (Peñalver, 1996d). Asimismo, con las familias Torymidae, Trichogrammatidae, Megaspilidae y Proctotrupidae pertenecientes al orden Hymenoptera (Anento et al., 1997). Además, han sido estudiadas hojas vegetales de varios géneros con marcas de herbivorismo producidas por orugas de lepidópteros, observadas en ejemplares de los afloramientos de Alto de la Venta y Río 
Rubielos (Peñalver, 1997; Peñalver y Martínez-Delclòs, 1997).

En las ritmitas bituminosas del Mioceno Superior lacustre (Vallesiense, biozonas de Mein MN9-MN10) de la Cuenca de Bicorp se han hallado recientemente ejemplares de tisanópteros, un heteróptero indeterminado, un ejemplar de coleóptero Curculionoidea (Lám. I, fig. 6) y numerosos ejemplares de pupas de dípteros de la familia Chironomidae y restos de sus exuvias.

En el Vallesiense (Mioceno Superior) de Libros han sido citados estadios larvarios de odonatos, coleópteros Curculionidae y un posible díptero (Navás, 1922; Fernández-Marrón, 1972, Peñalver, 1996b).

La primera cita de insectos fósiles en el Vallesiense (Mioceno Superior) de Bellver de Cerdanya es la de Villalta y Crusafont (1945). El área incluye un gran número de afloramientos, como Coll de Saig, Baltarga, Torrent de Vilella, Santa Eugenia, Beders, Barranco de Salanca, etc. El conjunto paleoentomológico se resume en la Tabla 5. En varios de estos afloramientos han sido descritas agallas, evidencias de actividad de puestas de insectos sobre hojas. Villalta (1956) estudió una agalla sobre una hoja de Fagus, presumiblemente producida por un díptero de la familia Cecidomydidae, para la que describió la especie Mikiola ? pontiensis Villalta, 1956. Diéguez et al. (1996) citan cinco tipos diferentes de agallas en hojas de Fagus y Quercus, atribuidas a dípteros e himenópteros: Mikiola pontiensis y Contarinia sp. (Diptera: Cecidomyiidae), y Neuropterus sp. (Hymenoptera: Cynipidae). Otras dos formas de agallas atribuidas una a dípteros sobre una Magnoliophyta y otra a himenópteros sobre Quercus no han podido ser emplazadas taxonómicamente.

En las diatomitas del Turoliense superior (Mioceno Superior) de Elche de la Sierra ha sido hallada una larva de odonato Anisoptera, posiblemente un Aeschnidae, juntamente con abundante paleoflora y paleofauna (e.g. Calvo et al., 1978). El ejemplar de odonato está depositado en la Colección museográfica del Grupo Cultural Paleontológico de Elche (Alicante).

En el yacimiento del Mioceno de la Cuenca de Sorbas (Sendra, com. personal, 1997) han aparecido acumulaciones masivas monoespecíficas de larvas de odonatos anisópteros (Lám. I, fig. 7). Algunas muestras están depositadas en el Museo del Departamento de Geología de la Universitat de València.

Por último, para el Mioceno, en la Cuenca del Guadiana han sido citadas galerías atribuidas a la posible actuación de dípteros quironómidos (Armenteros et al. 1986).

\section{Plioceno}

Existe un solo yacimiento de edad pliocena, localizado en Cuevas del Almanzora (Almería). Pertenece a la Cuenca de Vera y se han citado recientemente restos de insectos (Sendra, 1995); uno de ellos corresponde a un himenóptero adulto indeterminado.

\section{Cuaternario}

En el Pleistoceno Inferior de Venta Micena (Orce,

\begin{tabular}{|c|c|c|}
\hline ORDEN & Familia & Género y especie \\
\hline \multirow[t]{5}{*}{ ODONATA } & Sieblosiidae & Stenolestes hispanicus ${ }^{(1)}$ \\
\hline & Lestidae & Lestes sp. ${ }^{(2 \mathrm{y} 3)}$ \\
\hline & $\begin{array}{l}\text { Megapodagrionidae } \\
\text { / Pseudolestidae? }\end{array}$ & Cerdanyagrion miocenicus ${ }^{(4)}$ \\
\hline & Aeshnidae & Aeshna cerdanica $^{(5)}$ \\
\hline & Libellulidae & Trithemis pseudodistanti ${ }^{(6)}$ \\
\hline ISOPTERA & Hodotermitidae & Ulmeriella sp. ${ }^{(7)}$ \\
\hline HEMIPTERA & Pentatomidae & Pentatoma $?^{(3)}$ \\
\hline RAPHIDIOPTERA & Inocelliidae & Miofibla cerdanica $^{(8)}$ \\
\hline \multirow[t]{7}{*}{ COLEOPTERA } & Buprestidae & Chalcophora espanoli $^{(9)}$ \\
\hline & Coccinellidae & Scymninae gen. et sp. indet. ${ }^{(10)}$ \\
\hline & Carabidae & Campalita deplanatum $^{(9)}$ \\
\hline & Staphylinidae & Gen. et sp. indet. ${ }^{(10)}$ \\
\hline & Tenebrionidae & Gen. et sp. indet. ${ }^{(9 y 10)}$ \\
\hline & Curculionidae & Gen. et sp. indet. ${ }^{(9)}$ \\
\hline & Aphodinidae & Gen. et sp. indet. ${ }^{(9)}$ \\
\hline \multirow[t]{8}{*}{ HYMENOPTERA } & Ichneumonidae & Pigmaeolus cf. nitidus ${ }^{(11)}$ \\
\hline & Apidae & Apis species $\mathrm{F}^{(12)}$ \\
\hline & Vespidae & Vespula sp. ${ }^{(11)}$ \\
\hline & \multirow[t]{4}{*}{ Formicidae } & Messor sp. ${ }^{(11)}$ \\
\hline & & Liometopum sp. ${ }^{(11)}$ \\
\hline & & Lasius o Formica? ${ }^{(11)}$ \\
\hline & & Camponotus sp. ${ }^{(11)}$ \\
\hline & Scolidae & Gen. et sp. indet. ${ }^{(3)}$ \\
\hline TRICHOPTERA & Phryganeidae & Phryganea sp. ${ }^{(2 y 3)}$ \\
\hline \multirow[t]{4}{*}{ DIPTERA } & \multirow[t]{3}{*}{ Bibionidae } & Bibio sp. ${ }^{(13)}$ \\
\hline & & Plecia sp. ${ }^{(13)}$ \\
\hline & & Penthetria sp. ${ }^{(13)}$ \\
\hline & Mycetophilidae & Exechia sp. ${ }^{(13)}$ \\
\hline
\end{tabular}

Tabla 5. Registro, no icnológico, de insectos en los afloramientos del Mioceno Superior de Bellver de Cerdanya (Lleida). 1) Nel (1991a); 2) Arillo y Bremond (1992a); 3) Villalta y Crusafont (1945); 4) $\mathrm{Nel}$ et al. (1996); 5) Nel et al. (1994); 6) Nel (1991b); 7) Nel y Paicheler (1993); 8) Nel (1993); 9) Villalta (1962); 10) Ortuño y Arillo (1995); 11) Arillo y Bremond (1992b); 12) Nel et al. (1999) y 13) Arillo et al. (1992).

Granada), ciertas perforaciones en huesos de macromamíferos se han atribuido a larvas de insectos (Gibert y Caporicci, 1989).

En el Pleistoceno Superior de las Islas Canarias se 
encontraron estructuras atribuidas a celdas de nidificación de abejas solitarias, posiblemente de la familia Anthophoridae (Ellis y Ellis-Adams, 1993). Estas celdas de nidificación fósiles (Lám. I, fig. 8) han aparecido en varios afloramientos de la isla de Fuerteventura denominados Corralejo, El Quemado, Istmo de la Pared y Barranco de los Molinos (Rothe, 1986; Petit-Maire et al., 1987; Ellis y Ellis-Adams, 1993), pero también en la isla de Lanzarote, en El Jable, cerca de Tinajo (Ellis y EllisAdams, op. cit.). Idénticas celdas de nidificación han sido observadas por uno de nosotros (A.A.) en Maspalomas, Gran Canaria.

\section{CONSIDERACIONES FINALES}

Los yacimientos con insectos fósiles en España son escasos y han sido poco o nada estudiados, excepto una minoría de ellos. En una gran parte de la Península Ibérica (ver Figura 1), que incluye todo el oeste, la zona centro y prácticamente todo el sur, no se conocen yacimientos de este tipo. Si se compara el número de estos yacimientos con los conocidos en el resto de Europa, por ejemplo Francia, se observa una importante deficiencia. No ha existido en España, hasta los últimos años, un equipo que estudiase los insectos fósiles. No obstante, en la última década existe un importante impulso de estos estudios, lo que ha supuesto el descubrimiento de un gran número de yacimientos y el conocimiento con detalle de algunos de ellos, aunque dichos estudios están centrados en el Cretácico y en el Neógeno.

\section{AGRADECIMIENTOS}

Los autores agradecen especialmente la colaboración del Dr. Robert Wagner (Córdoba) por su aportación a las dataciones y localizaciones de los yacimientos del Carbonífero y Pérmico. Asimismo, al Dr. Rodrigo Soler (Madrid), Enrique Bernárdez (Oviedo), Dr. Paul Palmqvist (Málaga), Dr. Bienvenido Martínez, Dr. André Nel (París), Dr. Vladimir Zherikhin, Dra. N. Sinitchenkova, Dr. Alexander Ponomarenko y Dr. Alexander Rasnitsyn (Moscú), Dra. María Luisa Martínez-Chacón, Dr. Sixto Fernández y la Dra. M.Teresa Fernádez-Marrón, por sus consideraciones y aportaciones. A Armando Díaz-Romeral, Joaquín Sendra y a Rosa Martínez por prestarnos algunos ejemplares citados y figurados en este trabajo. A Manuel Iñesta, José Manuel Marín, Miguel Ángel Prieto, Dr. Carlos Santisteban, Dr. Plinio Montoya, José Ignacio Ruiz Omeñaca y Bienvenido Diez por su colaboración. A los Sres. Federico Górriz y Federico Alegre por su ayuda en la localización de los yacimientos de ámbar de Teruel. Este artículo es en parte fruto de una beca de la European Science Foundation, dentro del Network Fossil Insects, a uno de nosotros (X.M.D.) y se enmarca dentro del proyecto PB970061 de la DGES.

\section{BIBLIOGRAFÍA}

Alekseev, A. V. 1993. Jurassic and Lower Cretaceous Buprestidae (Coleoptera) from Eurasia. Paleontological Journal, 27, 9-34.

Alonso, J., Arillo, A., Barrón, E., Corral, C., Grimalt, J., López, J.F., López, R., Martínez-Delclòs, X., Ortuño, V., Peñalver, E., and Trinçao, P. (en prensa). A new fossil resin with biological inclusions in Lower Cretaceous Deposits from Álava (Northern Spain, BasqueCantabrian Basin). Journal of Paleontology.

Álvarez-Ramis, C. 1990. Présence de restes de Blattidae dans le faisceau Carrasconde du Bassin Stéphanien de Villablino (Léon, Espagne). Bulletin Société Histoire naturelle Autun, 131, 7-10.

Amerom, H.W.J. van. 1966. Phagophytichnus ekowskii nov. ichnogen. \& nov. ichnosp., eine missbildung infolge von insektenfrass, aus dem Spanischen Stephanien (Provinz León). Leidse Geologische Mededelinge, 38, 181-184.

Anadón, P. 1973. Estudio estratigráfico y sedimentológico de los afloramientos terciarios de Campins (Barcelona). Tesis de Licenciatura inédita. Universidad de Barcelona, $123 \mathrm{pp}$.

Anento, J.L., Peñalver, E. y Selfa, J. 1997. Un viaje al pasado: los himenópteros fósiles de Rubielos de Mora. Quercus, 140, 10-16.

Ansorge, J. 1991a. Zur Sedimentologie und Paläontologie des unterkretazischen Plattenkalkaufschlusses "La Cabrua" (Sierra del Montsec; Provinz Lerida/NE Spanien) unter besonderer Berücksichtigung der fossilen Insekten. Diplomarbeit, Ernst-Moritz-Arndt Universität Greifswald, Greifswald, 83 pp.

Ansorge, J. 1991b. Locustopsis africanus n. sp. (Saltatoria, Caelifera) aus der Unterkreide SW-Ägyptens. Neues Jarbuch für Geologie und Paläontologie, Mh. 4, 205212.

Ansorge, J. 1993. Bemerkenswerte Lebensspuren und ?Cretosphex catalunicus n.sp. (Insecta, Hymenoptera) aus den unterkretazischen Plattenkalken der Sierra del Montsec (Provinz Lérida, NE-Spanie). Neues Jarbuch für Geologie und Paläontologie, Abh. 190 (1), 19-35.

Ansorge, J. 1997. Insekten aus dem Buntsandstein (Untere Trias) von Mallorca (Spanien). IV Fachgespräch "Fossile Insekten", ClausthalZellerfeld. Juni. Abstract, 28-29.

Arbizu, M., Bernárdez, E., Peñalver-Mollá, E. y Prieto, M.A. 1998. El ámbar de Asturias. Abstracts del Congreso Mundial sobre Inclusiones en Ámbar, Vitoria, 147.

Arillo, A. 1994. Nota sobre una larva de Odonato del Oligoceno de Izarra (Álava, España) en la colección del Museo Geominero (Odonata, Anisoptera, Libellulidae). Boletín Geológico y Minero, 105, 325-328.

Arillo,A. 1998. Dípteros del ámbar de Álava. Valoración preliminar del material estudiado. Abstracts del Congreso Mundial sobre Inclusiones en Ámbar, Vitoria, 117.

Arillo, A. y Bremond, C. S. 1992a. Nota sobre la presencia de un Tricóptero y un Odonato en el Mioceno Superior de la depresión ceretana. Boletín Geológico y Minero, 103, 16-20. 
Arillo, A. y Bremond, C. S. 1992b. La entomofauna fósil del Mioceno superior de la depresión ceretana. Himenópteros, Graellsia, 48, 99-107.

Arillo, A. and Ortuño, V.M. 1997a. First record of the families Anthicidae and Chrysomelidae from the Oligocene of Izarra (Álava, Spain). The Coleopterists Bulletin, 51, 293-297.

Arillo, A. and Ortuño, V.M. 1997b. The fossil Acrididae (Orthoptera, Caelifera) from the Oligocene of Izarra (Álava, Spain). The antiquity of gregarious behavior. Geobios, 30, 231-234.

Arillo, A., Bremond, C. S. y Barrón, E. 1992. La entomofauna fósil del Mioceno superior de la depresión ceretana. Dípteros. Historia Natural'91 (Ed. A. Alemany), 553-561.

Arillo, A., Nel. A. and Ortuño, V.M. 1996. Two fossil bees from the Oligocene of Izarra (Álava, Spain) (Hymenoptera, Apoidea). Bulletin de la Société entomologique de France, 101, 59-64.

Armenteros, I., Dabrio, C., Alonso, G., Jorquera, A. y Villalobos, M. 1986. Laminación y bioturbación en carbonatos lagunares: Interpretación genética (Cuenca del Guadiana, Badajoz). Estudios Geológicos, 42, 271280.

Barrón, E., Ortuño, V.M. y Arillo, A. 1997. Estudio paleontológico del afloramiento mioceno de Izarra (Álava, España). Estudios del Museo de Ciencias Naturales de Álava, 12, 5-15.

Boscá, A. 1910. Cuenca calaminífera de Linares de Aragón. Asociación Española para el Progreso de la Ciencia, 4 (1 ${ }^{\mathrm{a}}$ parte), 171-181.

Brauckmann, C. 1993. Notiz über insekten-Reste aus dem Ober-Karbon in Spanien. Jahresberichte des Naturwissenschaftichen Vereins Wuppertal, 46, 115119.

Broutin, J. 1986. Étude paléobotanique et palynologique du passage carbonifère permien dans le sud-ouest de la Péninsule Ibérique. Cahiers de Paléontologie. Editions du Centre National de la Recherche Scientifique, Paris, $165 \mathrm{pp}$.

Calafat, F. 1988. Estratigrafía y Sedimentología de la litofacies Buntsandstein de Mallorca. Tesis de Licenciatura, Universitat de les Illes Balears, Palma de Mallorca, $126 \mathrm{pp}$.

Calafat, F. y Sáez, B. 1987. Paleofauna lacustre del Triásico inferior de Mallorca. $1^{e s}$ Jornades del Medi Ambient de Balears, Palma de Mallorca, 18-19.

Calvo, J.P., Elizaga, E., López-Martínez, N., Robles, F. y Usera, J. 1978. El Mioceno superior continental del Prebético Externo: Evolución del Estrecho Nordbético. Boletín Geológico y Minero, 89, 407-426.

Carpenter, F. M. 1963. A Megasecopteron from Upper Carboniferous strata in Spain. Psyche, 70, 44-49.

Castro, M. P. 1997. Huellas de actividad biológica sobre plantas del Estefaniense superior de La Magdalena (León, España). Revista Española de Paleontología, 12, 52-66.

Compte, A. 1995. Sobre la identidad del Odonato fósil Daemhippus cincuneguii (Gil Collado, 1926). Avances Entomología Ibérica, 1995, 319-332.
Diéguez, C., Nieves-Aldrey, J. L. and Barrón, E. 1996. Fossil galls (zoocecids) from the Upper Miocene of La Cerdaña (Lérida, Spain). Review of Palaeobotany and Palynology, 94, 329-343.

Díez, J. B., Canudo, J. I., Ferrer, J., Muñoz-Barragán, P., Ruíz-Omeñaca, J. I. y Soria, A. R. 1996. Transporte y resedimentación de troncos silicificados en el Albiense (Fm. Utrillas, Castellote, Cordillera Ibérica). Comunicaciones de la II Reunión de Tafonomía y Fosilización, Zaragoza, 97-102.

Ellis, W. N. and Ellis-Adams, A. 1993. Fossil brood cells of solitary bees on Fuerteventura and Lanzarote, Canary Islands (Hymenoptera: Apoidea). Entomologische Berichten Amsterdam, 53, 161-173.

Fernández-Marrón, M. T. 1972. Nota sobre la paleoecología del yacimiento de Libros (Teruel) en relación con los Artrópodos y las plantas fósiles del biotopo. Acta Geológica Hispánica, 7, 149-153.

Fernández-Marrón, M.T., Olive, A., Del Olmo, P. y Portero, J.M. 1979. La flora terciaria del yacimiento de Izarra (Álava) y sus implicaciones cronoestratigráficas. Boletín Geológico y Minero, 90, 6-12.

Fernández-Rubio, F. y Peñalver, E. 1994. Un nuevo ejemplar fósil de Zygaena? turolensis Fernández-Rubio, Peñalver y Martínez-Delclòs, 1991 (Lepidoptera; Zygaenidae). Estudios Museo Ciencias Naturales de Álava, 9, 39-48.

Fernández-Rubio, F., Peñalver, E. y Martínez-Delclòs, X. 1991. Zygaena? turolensis, una nueva especie de Lepidoptera Zygaenidae del Mioceno de Rubielos de Mora (Teruel). Descripción y filogenia. Estudios Museo Ciencias Naturales de Álava, 6, 77-93.

Gibert, J. y Caporicci, R. 1989. Tafonomía y paleoecología del yacimiento de Venta Micena. In: Los restos humanos de Orce y Cueva Victoria (Eds. J. Gibert Clols, D. Campillo Valero \& E. García Olivares). Institut Paleontològic Dr. M. Crusafont, 241-268.

Gil, J. 1926. Nota sobre algunos insectos fósiles de Ribesalbes (Castellón). Boletín del Instituto Geológico de España, 6, $3^{\mathrm{a}}$ serie, 89-107.

Gómez, J. E. 1979. Un ave y otras especies fósiles nuevas de la biofacies de Santa María de Meyá (Lérida). Boletín Geológico y Minero, 90, 333-346.

Gómez, J. E. 1984. Nuevos paleontínidos del yacimiento infracretácico de la "Pedrera de Meiá" (Lérida). Boletín Geológico y Minero, 95, 301-309.

Gómez, J. E. 1986. Nuevos insectos fósiles de las calizas litográficas del Cretácico Inferior del Montsec (Lérida). Boletín Geológico y Minero, 97, 717-736.

Iwaniw, E. 1985. Floral palaeoecology of debris flow dominated valley-fill deposits in the Lower Cantabrian of NE León, NW Spain. Anais Faculdade Ciências. Universidade do Porto, 64, supl. Papers on the Carboniferous of the Iberian Peninsula, 283-357.

Kukalová-Peck, J. and Brauckmann, C. 1992. Most Paleozoic Protorthoptera are ancestral hemipteroids: major wing braces as clues to a new phylogeny of Neoptera (Insecta). Canadian Journal of Zoology, 70, 2452-2473.

Lacasa, A. y Martínez-Delclòs, X. 1986a. Fauna y flora de 
los yacimientos Neocomienses del Montsec (Prov. Lérida). Paleontologia i Evolució, 20, 215-223.

Lacasa, A. y Martínez-Delclòs, X. 1986b. Meiatermes nuevo género fósil de insecto isóptero (Hodotermitidae) de las calizas neocomienses del Montsec (Provincia de Lérida, España). Institud d'Estudis Ilerdencs, Lérida, 65 pp.

Martínez-Delclòs, X. 1988. Els insectes. In: El Registre Fòssil (Eds. O. Riba i J. Gallemí). Història Natural dels Països Catalans, Barcelona, 15, 352-356.

Martínez-Delclòs, X. 1989a. Chresmoda aquatica n. sp. insecto Chresmodidae del Cretácico inferior de la sierra del Montsec (Lleida, España). Revista Española de Paleontología, 4, 67-74.

Martínez-Delclòs, X. 1989b. Ilerdaegomphus nom. nov. para el odonato adulto Palaeaeschna pallerolae del Cretácico Inferior del Montsec (Catalunya, España). Boletín Geológico y Minero, 100, 187-192.

Martínez-Delclòs, X. 1989c. Insectos del Cretácico inferior de las Hoyas (Cuenca). In: La fauna del pasado en Cuenca (Ed. Instituto Juan de Valdés). Actas del I Curso de Paleontología, Cuenca, 51-82.

Martínez-Delclòs, X. 1990. Insectos del Cretácico inferior de Santa Maria de Meià (Lleida): Colección Lluís Marià Vidal i Carreras. Treballs Museu Geologia Barcelona, 1, 91-116.

Martínez-Delclòs, X. 1991a. Insectes de les calcàries litogràfiques de la Serra del Montsec. Cretaci inferior de Catalunya, Espanya. In: Les calcàries litogràfiques del Cretaci del Montsec. Deu anys de campanyes paleontològiques (Ed. X. Martínez Delclòs). Institut d'Estudis Ilerdencs, Lleida, 91-110.

Martínez-Delclòs, X. 1991b. Insectes hemimetàbols del Cretaci inferior d'Espanya. Tafonomia $i$ Paleoautoecologia. Tesis Doctoral, Dept. G.D.G.P., Univ. de Barcelona, Barcelona, 784 pp.

Martínez-Delclòs, X. 1993. Blátidos (Insecta, Blattodea) del Cretácico inferior de España. Familias Mesoblattinidae, Blattulidae y Poliphagidae. Boletín Geológico y Minero, 104, 52-74.

Martínez-Delclòs, X. (en prensa). Paleoentomofauna del yacimiento de calizas litográficas de "Las Hoyas" (Cretácico inferior de Cuenca). In: "Las Hoyas", un lago de tiempos remotos (Ed. J.L. Sanz). Série Actas Académicas, 8, Cuenca.

Martínez-Delclòs, X. and Martinell, J. 1995. The oldest known record of social insects. Journal of Paleontology, 69, 594-599.

Martínez-Delclòs, X. et Nel, A. 1991. Découverte de trois insectes fossiles dans l'Oligocène inférieur du bassin de l'Ébre (Espagne) (Odonata, Lestidae, Aeshnidae). Bulletin du Museum national d'Histoire naturelle, Paris, 13, 157-165.

Martínez-Delclòs, X. y Nel, A. 1994. Los Gomphidae (Insecta, Odonata, Anisoptera) del Cretácico Inferior de España. Revista Española de Paleontología, 9, 176-184.

Martínez-Delclòs, X. and Nel, A. 1995a. Arthropods. In: Montsec \& Montral-Alcover. Two KonservatLagerstätten. Catalonia, Spain (Ed. X. Martínez Delclòs). II International Symposium on Lithographic Limestones. Institut d'Estudis Ilerdencs, Lleida, 39-46.
Martínez-Delclòs, X. and Nel, A. 1995b. Insects. In: Las Hoyas. A lacustrine Konservat-Lagerstätte. Cuenca, Spain (Ed. M. N. Meléndez). II International Symposium on Lithographic Limestones. Universidad Complutense de Madrid, Madrid, 36-41.

Martínez-Delclòs, X. et Ruiz de Loizaga, Ma . J. 1993. Les insectes des calcaires lithographiques du Crétacé inférieur d'Espagne. Faune et taphonomie. Geobios, M.S. 16, 195-201.

Martínez-Delclòs, X., Nel, A. and Popov, Y. A. 1995. Systematics and functional morphology of Iberonepa romerali $\mathrm{n}$. gen. and $\mathrm{n}$. sp., Belostomatidae from the spanish Lower Cretaceous (Insecta, Heteroptera). Journal of Paleontology, 69, 496-508.

Martínez-Delclòs, X., Peñalver, E. y Rasnitsyn, A. 1998. Los Hymenoptera del Ámbar del Cretácico Inferior de Álava (País Vasco, España). Abstracts del Congreso Mundial sobre Inclusiones en Ámbar, Vitoria, 119.

Meunier, F. 1902. Un nuevo cicádido del Kimeridgense de la Sierra del Montsech, provincia de Lérida (Cataluña). Memorias de la Real Academia de Ciencias y Artes Barcelona, 4, 269-275.

Meunier, F. 1903. Nuevas contribuciones a la fauna de los himenópteros fósiles. Memorias de la Real Academia de Ciencias y Artes Barcelona, 4, 461-465.

Meunier, F. 1904. Sur une cicadine du kiméridgien de la Sierra del Montsech (Catalogne). Feuille jeune Nature, 34, 119-121.

Meunier, F. 1914. Un blátido y una larva de odonato del Kimmeridgense de la Sierra del Montsech (Lérida). Memorias de la Real Academia de Ciencias y Artes Barcelona, 11, 121-126.

Montoya, P., Peñalver, E., Ruiz-Sánchez, F. J., Santisteban, C., Alcalá, L., Belinchón, M. y Lacomba, J. I. 1996. Los yacimientos paleontológicos de la cuenca terciaria continental de Rubielos de Mora (Aragón). Revista Española de Paleontología, $\mathbf{n}^{\circ}$ extra., 215-224.

Navás, L. 1922. Algunos fósiles de Libros (Teruel). Boletín de la Sociedad Ibérica de Ciencias Naturales, 11, 51-61.

Nel, A. 1991a. Description de quelques Sieblosiidae fossiles nouveaux (Odonata, Zygoptera, Lestoidea). Nouvelle Revue d'Entomologie (N.S), 8, 367-375.

$\mathrm{Nel}$, A. 1991b. Un nouvel odonate fossile du Miocène de Bellver de Cerdaña (Espagne) (Odonata, Libellulidae). Entomologica Gallica, 2, 129-130.

Nel, A. 1993. Nouveaux Raphidioptères fossiles du Cénozoïque de France et d'Espagne (Raphidioptera, Raphidiidae, Inocelliidae). E.P.H.E., Biologie et Evolution des Insectes, 6, 99-108.

Nel, A. et Arillo, A. 1995. Révision de Mastotermes haidingeri (Heer, 1849). Description de deux nouveaux Mastotermes de l'Oligocène de France et d'Espagne (Isoptera, Mastotermitidae). Bulletin de la Société entomologique de France, 100, 67-74.

Nel, A. et Martínez-Delclòs, X. 1993a. Essai de révision des Aeschnidioidea (Odonata, Anisoptera). Cahiers de Paléontologie, CNRS Ed., 99 pp.

Nel, A. y Martínez-Delclòs, X. 1993b. Nuevos Zygoptera y Anisoptera (Insecta: Odonata) en el Cretácico inferior de España. Estudios Geológicos, 49, 351-359. 
Nel, A. et Paicheler, J.-C. 1993. Les Isoptera Fossiles. État actuel des connaissances, implications paléoécologiques et paléoclimatologiques (Insecta, Dictyoptera). Cahiers de paléontologie, CNRS Ed., 101-179.

Nel, A., Martínez-Delclòs, X., Escuillé, F. et Brisac, P. 1994. Les Aeshnidae fossiles: Etat actuel de connaissances (Odonata, Anisoptera). Neues Jarbuch für Geologie und Paläontologie, Abh., 194, 143-186.

Nel, A., Arillo, A. et Ortuño, V. M. 1995. Découverte du premier Libellulidae Trameinae de 1'Oligocène d’Espagne (Odonata, Anisoptera). Bulletin de la Société entomologique de France, 100, 487-488.

Nel, A., Arillo, A. and Martínez-Delclòs, X. 1996. New fossil Odonata (Insecta) from the Upper Miocene of France and Spain (Anisoptera and Zygoptera). Neues Jarbuch für Geologie und Paläontologie, Abh., 199, 167219.

Nel, A., Arillo, A. and Ortuño, V. M. 1997. New Western Paleartic Cenozoic Odonata (Zygoptera and Anisoptera). Bulletin de la Société entomologique de France, 102, 265-270.

Nel, A., Martínez-Delclòs, X., Arillo, A. and Peñalver, E. 1999. The fossil Apis Linné, 1758 (Hymenoptera, Apidae). Palaeontology, 42, 243-285.

Ortuño,V. M. 1998. Artropodofauna fósil del yacimiento de Ámbar Alavés (Cretácico inferior). Abstracts del Congreso Mundial sobre Inclusiones en Ámbar, Vitoria, 115.

Ortuño, V. M. y Arillo, A. 1995. La entomofauna del Mioceno Superior de la depresión ceretana (Lleida, NE de España). Coleópteros I. Boletín Geológico y Minero, 106, 407-414.

Ortuño, V.M. and Arillo, A. 1997a. Discovery of the genus Ernobius THOMSON (Coleoptera: Anobiidae) from the Oligocene of Izarra, Álava, Spain. The Coleopterist Bulletin, 51, 71-73.

Ortuño, V.M. y Arillo, A. 1997b. Nuevos Heteroptera del Mioceno de Izarra (Álava, España). Estudios del Museo de Ciencias Naturales de Álava, 12, 17-23.

Ortuño, V.M. y Arillo, A. 1997c. Presencia del género Geocoris en el Oligoceno de Izarra (Álava, España). Descripción de Geocoris monserrati sp. nov. (Heteroptera, Lygaeidae). Nouvelle revue d'Entomologie, 14, 359-363.

Ortuño, V.M. y Arillo, A. 1997d. Primer dato de un estadío preimaginal de Colymbetinae (Coleoptera, Dytiscidae) del Oligoceno de Izarra (Álava, España) (Coleoptera, Dytiscidae). Nouvelle revue d'Entomologie, 14, 141-145.

Ortuño, V. M., Arillo, A., Barrón, E. y Alonso, J. 1996. Primeros datos sobre el paleoecosistema del lago Oligoceno de Izarra (Álava). Resúmenes de Ponencias y Comunicaciones, 125 Aniversario-XII Bienal de la RSEHN, Madrid, p. 196.

Peñalver, E. 1996a. Larvas de Stratiomyidae (Insecta: Diptera) en el Mioceno lacustre de Ribesalbes (Castellón). Comunicaciones XII Jornadas de Paleontología, Badajoz, 95-97.

Peñalver, E. 1996b. Los yacimientos con insectos fósiles de Aragón (España). Boletín Sociedad Entomológica Aragonesa, 16, 139-146.
Peñalver, E. 1996c. Presencia del género Penthetria (Insecta: Diptera: Bibionidae) en el Mioceno lacustre de Rubielos de Mora y Ribesalbes. Comunicaciones XII Jornadas de Paleontología, Badajoz, 97-99.

Peñalver, E. 1996d. Insectos fósiles en el Mioceno de Rubielos de Mora (Teruel). Memoria de la excavación paleontológica de septiembre de 1994. Teruel, 83-84, (1992-1996), 157-204.

Peñalver, E. 1997. Hojas fósiles del Terciario de Teruel con marcas de herbivorismo debidas a orugas. Boletín Sociedad Entomológica Aragonesa, 19, 29-33.

Peñalver, E. y Martínez-Delclòs, X. 1993. Aportaciones a la paleoentomofauna del Mioceno inferior de Ribesalbes (Castellón, España). Comunicaciones IX Jornadas de Paleontología, Málaga, 85-90.

Peñalver, E. y Martínez-Delclòs, X. 1996. Niveles con concentraciones de insectos chironómidos en el Mioceno de Rubielos de Mora (Teruel) y Ribesalbes (Castellón): consideraciones tafonómicas y paleoecológicas. Comunicaciones de la II Reunión de Tafonomía y Fosilización, Zaragoza, 305-310.

Peñalver, E. y Martínez-Delclòs, X. 1997. Evidencias de interacción entre insectos y plantas durante el Mioceno (cuencas lacustres de Rubielos de Mora, Teruel y Ribesalbes-Alcora, Castellón), In: Avances en el conocimiento del Terciario Ibérico (Eds. J. P. Calvo y J. Morales). Madrid, 149-152.

Peñalver, E., Nel, A. and Martínez-Delclòs, X. 1995. New Nothochrysinae from the spanish Miocene (Neuroptera, Chrysopidae). Bulletin de la Société entomologique de France, 100, 481-487.

Peñalver, E., Nel, A. y Martínez-Delclòs, X. 1996. Insectos del Mioceno inferior de Ribesalbes (Castellón, España). Paleoptera y Neoptera poli- y paraneoptera. Treballs del Museu de Geologia de Barcelona, 5, 15-95.

Petit-Maire, N., Rosso, J. C., Delibrias, G., Meco, J. et Pomel, S. 1987. Paléoclimats de 1'île de Fuerteventura (Archipel Canarien). Palaeoecology of Africa and the surrounding islands, 18, 351-356.

Rasnitsyn, A. 1975. Hymenoptera Apocrita of Mesozoic. Akademiia Nauk, CCCP, Trudy Paleontologischeskogo Instituta, Moscú, 174, 135 pp.

Rothe, P. 1986. Kanarische Inseln. Sammlung geologische Führer, 81, 226 pp.

Schneider, J. 1983. Die Blattodea (Insecta) des Paläozoikums. Teil 1: Systematik, Ökologie und Biostratigraphie. Freiberger Forschungsheft, Leipzig, C 382, 106-145.

Sendra, J. 1995. El Konservat Fossil-Lagerstätten de Cuevas del Almanzora (Almería, España). Extended abstracts II International Symposium on Lithographic Limestones, Cuenca, 133-137.

Shcherbakov, D.E., Lukashevich, E.D. and Blagoderov, V.A. 1995. Triassic Diptera and initial radiation of the order. International Journal of Dipterological Research, 6, 75-115.

SolerGijón, R. 1997. Euselachian sharks from the late Carboniferous of the Puertollano Basin, Spain: Biostratigraphic and palaeoenvironmental implications. Modern Geology, 21, 137-169. 
Vía, L. y Calzada, S. 1985. Artrópodos fósiles del Triásico de Alcover-Montral. (I-Insectos). Resúmenes del II Coloquio de Estratigrafía y Paleogeografía del Pérmico y Triásico de España, La Seu d'Urgell, p. 119.

Vía, L. y Calzada, S. 1987. Artrópodos fósiles triásicos de Alcover-Montral (I. Insectos). Cuadernos de Geología Ibérica, 11, 273-280.

Vía, L., Villalta, J. F. y Esteban, M. 1977. Paleontología y paleoecología de los yacimientos fosilíferos del Muschelkalk superior entre Alcover y Mont-ral (Montañas de Prades, provincia de Tarragona). Cuadernos Geología Ibérica, 4, 247-256.

Vigón, M. 1980. Nuevas aportaciones al estudio paleoecológico del yacimiento Mioceno de Ribesalbes. Tesis de Licenciatura, Universidad Complutense, Madrid, $66 \mathrm{pp}$.

Vigón, M. A. 1981. Aportación al conocimiento de la paleoecología del yacimiento mioceno de Ribesalbes. Coloquios de Paleontología, 36, 61-66.

Villalta, J. F. 1956. Dos zoocecidías fósiles del Mioceno de
Cerdaña (prov. de Lérida). Cursos y Conferencias del Instituto Lucas Mallada (I Reunión del Terciario), Sabadell, 63-64.

Villalta, J. F. 1962. Dos coleópteros fósiles procedentes de la depresión de Cerdanya (Lérida). Estudios Geológicos, 18, 105-109.

Villalta, J. F. y Crusafont, M. 1945. La flora miocénica de la depresión de Bellver. Ilerda, 3, 339-353.

Wagner, R. H y Artieda, J. I. 1970. La Cuenca minera Ciñera-Matallana. S.A. Hullera Vasco-Leonesa, Litografía Everest, León, 288 pp.

Whalley, P. E. and Jarzembowski, E. A. 1985. Fossil insects from the lithographic limestone of Montsech (late Jurassic-early Cretaceous), Lérida Province, Spain. Bulletin of the British Museum Natural History (Geology), 38, 381-412.

Zherikhin, V.V. and Gratshev, V.G. 1997. The Early Cretaceous weevils from Sierra del Montsec, Spain (Insecta: Coleoptera: Curculionoidea). Cretaceous Research, 18, 625-632. 